Cahiers "Mondes anciens »

MONDES

ANCIENS

Histoire et anthropologie des mondes anciens

3 | 2012

Femmes de paroles

\title{
Quand une chrétienne prend la parole
}

Monique dans le De ordine et le De beata uita d'Augustin

\section{Mickaël Ribreau}

\section{(2) OpenEdition}

Journals

Édition électronique

URL : http://journals.openedition.org/mondesanciens/773

DOI : $10.4000 /$ mondesanciens. 773

ISSN : 2107-0199

Éditeur

UMR 8210 Anthropologie et Histoire des Mondes Antiques

Référence électronique

Mickaël Ribreau, "Quand une chrétienne prend la parole », Cahiers « Mondes anciens » [En ligne],

3 | 2012, mis en ligne le 23 mai 2012, consulté le 19 avril 2019. URL : http://journals.openedition.org/ mondesanciens/773 ; DOI : 10.4000/mondesanciens.773

Ce document a été généré automatiquement le 19 avril 2019

\section{(c) (i) (9)}

Les Cahiers «Mondes Anciens » sont mis à disposition selon les termes de la licence Creative Commons Attribution - Pas d'Utilisation Commerciale - Pas de Modification 4.0 International. 


\title{
Quand une chrétienne prend la parole
}

\author{
Monique dans le De ordine et le De beata uita d'Augustin ${ }^{1}$
}

\author{
Mickaël Ribreau
}

1 «Prends, lis $»^{2}$. Ces mots célèbres, entendus par Augustin, dans un jardin à Milan à la fin du mois d'août 386, conduisent le futur évêque d'Hippone à lire un passage de Paul. Augustin décide alors de se convertir au christianisme et de devenir candidat au baptême, qu'il reçoit durant la veillée pascale de 387. Dans l'intervalle, à cause de problèmes pulmonaires $^{3}$ et de sa conversion, Augustin abandonne son poste de professeur de rhétorique et se retire dans les environs de Milan, à Cassiciacum, dans la maison de campagne d'un grammairien milanais, Verecundus ${ }^{4}$. Accompagné de membres de sa famille, sa mère Monique, son frère Navigius, son fils Adéodat, ses cousins, Lartidianus et Rusticus, de son ami Alypius et de ses élèves et cousins africains Licentius et Trygetius ${ }^{5}$, Augustin s'adonne à la lecture des Psaumes $^{6}$ et à la composition de ses premières œuvres conservées : trois dialogues philosophiques, le Contra Academicos, le De beata vita, le De ordine, et les Soliloques ${ }^{7}$. Les trois dialogues sont présentés comme le compte rendu écrit de discussions réelles, prises en note par des sténographes, notarii, et s'enchaînent, comme le montrent plusieurs rappels d'une œuvre à l'autre. Dans la communauté de Cassiciacum se trouve une femme, Monique, la mère d'Augustin ${ }^{8}$. Dans le premier dialogue, le Contra academicos, qui réunit Augustin, ses deux élèves et son frère, Monique est présentée comme la maîtresse de maison qui vient mettre un terme aux discussions philosophiques, invitant son fils et ses amis à passer à table ${ }^{9}$. En revanche, dans les deux œuvres suivantes, elle prend une part active au dialogue et, tout en soulignant sa position particulière, voire inédite, de femme dans un dialogue philosophique, elle prend la parole à l'invitation de son fils. La critique a depuis longtemps souligné l'originalité de cette prise de parole, en s'attachant en particulier à la question de l'historicité des dialogues ${ }^{10}$ : une femme africaine d'origine relativement modeste, comme Monique, pouvait-elle tenir les discours qui lui sont prêtés dans le De beata uita ${ }^{11}$ et le De ordine ${ }^{12}$ ? D'autres critiques ont davantage souligné le rôle de Monique dans la conversion d'Augustin en étudiant les dialogues comme une préparation à la "vision d'Ostie ", extase mystique partagée entre Monique et Augustin et narrée dans les Confessions ${ }^{13}$. Cependant la mise en scène de la 
prise de parole de Monique a été rarement analysée avec précision ${ }^{14}$. Augustin met-il en valeur sa parole de femme? Comment est-elle caractérisée ? Comment s'articule-t-elle avec celle des autres participants de la discussion philosophique? Quel statut Monique at-elle dans ce dialogue essentiellement masculin? Et comment expliquer ou interpréter ce statut?

2 Nous laisserons de côté la question de l'historicité des dialogues ${ }^{15}$. Ce qui compte n'est pas de savoir si Monique a réellement tenu les propos rapportés dans les deux dialogues, si elle maîtrisait tel ou tel savoir qui lui est attribué, mais comment Augustin a décidé de mettre en scène sa mère, une chrétienne, dans ses œuvres, écrites dans l'attente du baptême. Les deux dialogues s'organisant de la même manière, ou de façon complémentaire, nous les aborderons ensemble, pour voir tout d'abord comment la parole de la femme qu'est Monique est justifiée, puis comment elle s'insère dans l'économie d'ensemble des dialogues, enfin en quoi son appartenance à la religion chrétienne est décisive.

\section{Une parole qui ne va pas de soi}

\section{Une parole justifiée aux seuils des dialogues}

3 Le 13 novembre 386, pour fêter son anniversaire, Augustin offre à ses invités un repas de fête à la fois matériel et spirituel. Il propose à ses convives des discussions philosophiques qui nourriront leur âme et dont le thème est le bonheur. De même que chez Cicéron, le dialogue est précédé d'une préface, dans laquelle sont précisés l'occasion du dialogue, le lieu et les protagonistes :

Autem, non enim uereor eos singulari benignitati tuae notos interim nominibus facere, in primis nostra mater, cuius meriti credo esse omne quod uiuo; Nauigius frater meus, Trygetius et Licentius, ciues et discipuli mei; nec Lastidianum et Rusticum consobrinos meos, quamuis nullum uel grammaticum passi sint, deesse uolui, ipsumque eorum sensum communem, ad rem quam moliebar, necessarium putaui. Erat etiam nobiscum aetate minimus omnium, sed cuius ingenium, si amore non fallor, magnum quiddam pollicetur, Adeodatus filius meus.

(De beata uita, 1, 6, BA 4/1, p. 63)

Il y avait - je ne crains pas de les faire connaître à ton insigne bienveillance par leur nom pour l'instant - en premier lieu notre mère, à qui appartient, j'en ai la conviction, tout le mérite de ma vie, Navigius, mon frère, Trygetius et Licentius, concitoyens et élèves ; aussi mes cousins, Lartidianus et Rusticus, bien qu'ils n'aient même pas été soumis au grammairien, je n'ai pas voulu qu'ils fussent absents, et j'ai même pensé que l'opinion commune qui était la leur était nécessaire pour le projet que j'avais. Il y avait encore avec nous le plus jeune de tous, mais en possession d'une intelligence qui, si mon affection ne m'abuse, promet de grandes choses, mon fils Adéodat.

Dans cette préface, Augustin justifie auprès du dédicataire de son œuvre, Manlius Théodore ${ }^{16}$, ancien préfet du prétoire qui s'était retiré de la vie publique afin de se consacrer à la composition de divers ouvrages philosophiques, le choix des interlocuteurs, car ils ne correspondent guère aux protagonistes des dialogues cicéroniens - ou de l'Antiquité tardive, que ce soit l'Octavius de Minucius Felix ou les Saturnales de Macrobe -, qui sont d'ordinaire des membres de l'aristocratie romaine. Augustin commence par le personnage le plus surprenant, au regard de la tradition philosophique cicéronienne, une femme, Monique, dont il justifie la présence par la filiation et par le rôle qu'elle a joué dans sa formation spirituelle. Contrairement au Contra 
academicos, qui réunissait des lettrés, Augustin et ses élèves, le De beata uita présente une société plus mêlée, car le projet d'Augustin requiert «l'opinion commune", sensum communem. Par leur position dans la présentation, en première et en dernière place, deux personnages sont mis en valeur : Monique, mère d'Augustin, et Adéodat, son fils ${ }^{17}$.

De même, dans la préface du deuxième livre du De ordine, Augustin justifie la présence de Monique à l'attention du dédicataire de l'œuvre, le poète Zénobius ${ }^{18}$. Ce lettré pourrait être choqué qu'une femme prenne la parole. Augustin écrit alors :

Nobiscum erat etiam mater nostra, cuius ingenium atque in res diuinas inflammatum animum, cum antea conuictu diuturno et diligenti consideratione perspexeram, tum uero in quadam disputatione non paruae rei, quam die natali meo cum conuiuis habui atque in libellum contuli, tanta mihi mens eius apparuerat, ut nihil aptius uerae philosophiae uideretur. Itaque institueram, cum abundaret otio, agere ut colloquio nostro non deesset. Quod et in primo etiam huius operis libro abs te cognitum est.

(De ordine, II, 1, BA 4/2, p. 162-164)

Il y avait aussi avec nous notre mère, dont j'avais perçu les dispositions naturelles et l'esprit enflammé pour les choses de Dieu déjà auparavant grâce à une longue vie commune et à l'attention mise à l'observer ; mais surtout lors d'un certain débat sur un sujet important, débat que j'eus avec des convives le jour de mon anniversaire et que j'ai consigné dans un petit livre ; son intelligence m'avait paru si grande qu'elle me semblait mieux faite qu'aucune autre pour la vraie philosophie. C'est pourquoi j'avais décidé, comme elle avait une abondance de loisir, d'obtenir qu'elle ne fit pas défaut à notre entretien, ce dont tu t'es déjà rendu compte au livre premier de cet ouvrage.

6 Augustin justifie davantage que dans le dialogue précédent la présence de Monique. Les habitudes du genre, le dialogue philosophique, et le dédicataire expliquent également cette justification; Augustin renvoie par ailleurs au De beata uita, qui a créé un précédent. La justification de la participation de Monique semble d'autant plus nécessaire que, dans la fiction du dialogue, elle ne prend pas la parole dans le cadre festif de l'anniversaire de son fils, mais lors d'un jour ordinaire. Enfin, cette justification permet de mettre davantage en valeur la première prise de parole de Monique, présentée comme imprévue dans le premier livre du même dialogue. Dans cette première intervention, elle souligne en effet l'incongruité de sa présence.

\section{Une justification mise en scène dans le De ordine}

7 En effet, dans le deuxième entretien du livre I du De ordine, après un moment de tension, Monique entre dans la pièce où se trouvent Augustin et ses disciples; elle demande si le sujet de l'entretien, qu'elle connaissait, a bien avancé. À l'invitation d'Augustin, son entrée et sa question sont consignées par les notarii, qui avaient déjà pris en notes les propos d'Augustin et de ses élèves ${ }^{19}$. Monique s'écrie alors :

Quid agitis inquit? Numquidnam in illis quos legitis libris etiam feminas unquam audiui in hoc genus disputationis inductas.

(De ordine, I, 31, BA 4/2, p. 150)

Que faites-vous? Est-ce que dans ces livres que vous lisez, j'ai jamais appris que des femmes aussi aient été associées à une discussion de ce genre?

8 Augustin nous montre donc Monique pleinement consciente de l'incongruité de sa présence, non pas dans l'entretien, mais dans le livre qui le consigne. En effet, en Tusculanes, III, 15, 31, par exemple, Cicéron affirme que Socrate ne voyait Xanthippe qu'à son foyer ${ }^{20}$. La réaction de Monique prévient celle du lecteur, comme du poète Zénobius, auquel l'œuvre est adressée. 
9 Mais Augustin oppose à cette conception une autre vision de la tradition philosophique, qui accepte plus volontiers la parole féminine :

Curiosi uel nimium studiosi contempta uilitate liminis intrare perrexerint, me tecum philosophantem non moleste ferent nec quemquam istorum quorum meis litteris sermo miscetur, fortasse contemnent. (...) Doctissimorum autem hominum litterae etiam sutores philosophatos et multo uiliora fortunarum genera continent: qui tamen tanta ingenii uirtutisque luce fulserunt, ut bona sua cum qualibet huiuscemodi nobilitate nullo modo uellent, etiamsi possent, ulla conditione mutare. Nec deerit, mihi crede, tale hominum genus cui plus placeat hoc ipsum quia mecum philosopharis, quam si quid hic aliud aut iucunditatis aut grauitatis inuenerit. Nam et feminae sunt apud ueteres philosophatae et philosophia tua mihi plurimum placet.

(De ordine, I, 31, BA 4/2, p. 152)

mais si [des lecteurs], curieux ou extrêmement studieux, sans tenir compte de la banalité de l'entrée en matière, persistent à y pénétrer, ils ne trouveront pas mauvais que je philosophe avec toi et ils ne mépriseront peut-être aucun de ceux dont les propos sont mêlés à mes écrits. (...) Et même les écrits des hommes les plus savants font place à des savetiers qui philosophent et à des conditions d'un niveau bien plus bas, qui ont cependant brillé d'une telle lumière par l'esprit et la vertu qu'à aucun prix ils ne voudraient, même s'ils le pouvaient, échanger leurs biens contre cette espèce de notoriété, quelle qu'elle soit. Et il ne manquera pas, croismoi, d'hommes de cette sorte, pour aimer mieux même ce détail que tu philosophes avec moi plutôt que de trouver ici quelque autre trait plaisant ou grave. Car des femmes aussi ont philosophé chez les anciens et ta philosophie me plaît infiniment.

10 De même que dans le De beata uita, ce qui pose problème est le fait que Monique soit une femme, mais surtout qu'elle ne soit pas lettrée ${ }^{21}$, comme le montre le parallèle avec les savetiers ${ }^{22}$; Augustin, opposant culture et pratique de vie, met en valeur la lumière de l'esprit et la vertu d'un mode de vie. C'est ce dernier qui peut justifier que Monique soit une philosophe. De plus, Augustin renvoie sa mère et son lecteur à une autre tradition qui ne méprise pas les femmes en philosophie; peut-être Augustin songe-t-il à Diotime, à la mère d'Hortensius, à Helvia la mère de Sénèque ou à Marcella la mère de Porphyre ${ }^{23}$. Augustin ne donne pas de nom. La tradition philosophique ne semble pas être le réel problème. Le point essentiel en revanche est la présentation de Monique comme une philosophe, qui possède sa philosophia.

11 Ces trois passages nous montrent donc que la parole d'une femme, surtout d'une femme sans culture, comme Monique, n'allait pas de soi et qu'il convenait pour Augustin de la justifier. En fait, il la justifie en tenant compte du genre dans lequel s'inscrivent le De beata uita et le De ordine, des dédicataires des œuvres, deux lettrés, ainsi, sans doute, que $\mathrm{du}$ lectorat visé, un public lettré, par l'intermédiaire des deux dédicataires. Mais comment la parole de Monique s'articule-t-elle avec celle des autres protagonistes des dialogues?

\section{Une parole libre : un second maître de parole}

\section{Une parole libre et décisive}

Deux éléments caractérisent les interventions de Monique : leur liberté et leur effet dans le débat. Dans les deux dialogues, Augustin est le maître du jeu ${ }^{24}$. Dans le De beata uita, il le justifie par la célébration de son anniversaire ${ }^{25}$. C'est donc lui qui propose le sujet du débat : le bonheur ; c'est lui qui distribue la parole. Ainsi, alors que Licentius veut changer le sujet de la conversation, Augustin affirme : 
Inuita me, inquam, natali tuo, quando dignaberis; quidquid apposueris libenter sumam. Qua conditione hodie apud me ut epuleris peto, nec flagites quod fortasse non est paratum.

(De beata uita, 2, 10, BA 4/1, p. 70)

Invite-moi pour ton anniversaire : si tu me fais cet honneur, tout ce que tu mettras sur la table, je le prendrai volontiers. C'est avec ces dispositions que je te demande de prendre ton déjeuner aujourd'hui à ma table, sans réclamer ce qu'on n'a peut-

être pas préparé.

13 Augustin donne la parole, mais Monique n'a pas besoin de permission. De même dans le De ordine, contrairement aux autres convives, et ce de façon plus marquée que dans le De beata uita, Monique peut prendre la parole librement, alors que les autres protagonistes demandent à Augustin la permission de parler $^{26}$. De plus, Monique intervient à des moments présentés comme décisifs ; par exemple, dans le De ordine, avant son arrivée imprévue, les trois partenaires du dialogue, Licentius, Trygetius et Augustin ne parviennent pas à débattre comme le souhaiterait le maître de parole. Augustin rabroue Licentius ${ }^{27}$ et pleure devant l'incapacité de ses élèves à réfléchir ${ }^{28}$. Un peu plus loin dans ce même entretien, alors que les autres participants rient d'avoir été comme pris au piège par diverses concessions que tous, exceptée Monique, ont accordées, la mère d'Augustin "qui était restée longtemps sans réagir » demande à son fils de lui expliquer de façon claire ce qui lui a semblé bien entortillé et parvient alors à une conclusion frappante : nul ne peut parvenir à Dieu sans l'avoir cherché29. Puis, de même qu'un sourire d'Augustin entraîne une question de Monique, de même le sourire de cette dernière conduit un autre convive à poursuivre. Pour répondre à une hésitation de ce dernier, Monique prend à nouveau la parole, alors que le reste de l'assemblée demeure silencieuse.

Le quatrième et dernier entretien du De ordine va dans le même sens. Monique parle après un silence ou une hésitation; ses propos sont des charnières qui font avancer le débat ${ }^{30}$. Dans le dernier entretien du De beata uita, constitué d'un long monologue d'Augustin, ce dernier reprend les propos de Monique, énoncés dans l'entretien précédent. Ce qu'a affirmé Monique et que tous ont reconnu comme vrai est donc le point de départ nécessaire de l'entretien conclusif ${ }^{31}$. Ce qui nous invite à considérer comment la parole de Monique s'articule avec celle du maître du dialogue.

\section{Deux personnages doubles : Monique et Augustin ${ }^{32}$}

15 Au début du De beata uita, avant de proposer le sujet de la conversation proprement dite, le bonheur, Augustin distingue le corps de l'âme, qui tous deux doivent être nourris. Augustin affirme tout d'abord que l'homme est composé d'un corps et d'une âme. Navigius reconnaît son ignorance. S'ensuit une série de questions-réponses entre Augustin et son frère, auxquels s'ajoute Licentius. Après que l'auditoire a convenu que le corps avait besoin de nourriture pour croître ${ }^{33}$, Augustin demande si la nourriture de l'âme est la science. Monique répond: «Assurément, je crois que l'âme ne se nourrit que de l'intelligence et de la connaissance de choses ». Trygetius n'est pas entièrement convaincu et hésite. Monique répond alors d'elle-même. Alors que les convives expriment leurs doutes par des murmures, Augustin reprend la parole et reformule les propos de Monique en termes plus abstraits ${ }^{34}$ et déroule une série de questions-réponses. Cette première prise de parole de Monique est construite en parallèle avec celle d'Augustin : affirmation d'une idée, puis doute, hésitation émise par l'un des participants, puis questions-réponses pour convaincre. Cependant, ce n'est pas Monique qui mène à bien le troisième moment (les questions-réponses), mais Augustin, maître du repas pour l'âme qu'est le dialogue. Plus loin, Augustin et Trygétius dialoguent. Le débat est mené par le 
maitre. Or, Monique prend la parole et déplace quelque peu la question, permettant à Augustin de parvenir à une étape essentielle : est heureux celui qui a Dieu ${ }^{35}$. Pour ce faire, la mère et le fils procèdent par questions et réponses, par approbation successive. Mais qu'est-ce qu'avoir Dieu? Trois réponses sont données. La dernière est celle d'Adéodat ( « ne pas avoir l'esprit impur, voilà ce que signifie avoir Dieu »36). Monique approuve en particulier cette troisième définition ${ }^{37}$. Or, lors du second entretien, Augustin revient sur les trois définitions et s'arrête en particulier sur celle donnée par Adéodat, qui « a l'esprit plus candide et plus pur que les autres auditeurs $»^{38}$. La réaction de Monique préfigurait donc celle d'Augustin et attirait l'attention du lecteur-auditeur sur Adéodat.

Dans l'intermède du De beata uita, situé entre le premier et le deuxième entretien, le passage de parole entre Augustin et Monique est mis en scène. Augustin, son frère et ses disciples y reviennent sur un sujet qu'ils avaient abordé la veille, dans le Contra academicos I. Or les cousins, la mère et le fils d'Augustin, qui étaient alors absents, ne comprennent rien aux propos des autres convives sur les académiciens :

Qui mihi prorsus similes uisi sunt, quod plerumque solet, iis qui cum epulantur inter auidissimos rapacissimosque conuiuas, a rapiendo uel grauitate sese abstinent, uel pudore terrentur. Et quia ego inuitaueram (...), commouit me illa inaequalitas mensae nostrae et discrepantia. Arrisi matri. Atque illa liberrime quod minus habebant, quasi de suo cellario promendum imperans: iam dic nobis, inquit, et redde qui sint isti Academici, et quid sibi uelint? Cui breuiter cum exposuissem aperteque, ita ut nemo illorum ignarus abscederet: isti homines, inquit, caducarii sunt (quo nomine uulgo apud nos uocantur, quos comitialis morbus subuertit), et simul surrexit ut abiret; atque hic omnes laeti ac ridentes, interposito fine discessimus.

(De beata uita, 2, 16, BA 4/1, p. 86)

Ils me parurent ressembler tout à fait, c'est une scène que l'on voit souvent, à ces gens qui dînent au milieu de convives extrêmement avides et gloutons et qui s'abstiennent par gravité de rien ravir ou sont détournés de le faire par timidité. Et parce que c'est moi qui avais invité (...), j’ai été choqué de ce manque d'égalité et de cet accord faussé à notre table. Je souris à ma mère. Et elle, montrant la plus grande libéralité, comme si de son cellier à elle on devait tirer ce qu'ils avaient le moins, me donne cet ordre: dis-nous donc et donne-moi réponse: qui sont ces Académiciens et à quoi ils veulent en venir? Comme je lui avais fait un exposé bref et clair, de façon que personne ne partît sans les connaître, elle dit : « ces hommes sont épileptiques» (c'est le nom dont on désigne généralement parmi nous ceux que terrasse le haut mal) ; et en même temps elle se leva pour s'en aller. Et alors, un point final étant mis, nous nous retirâmes tous dans la joie de nos rires.

17 Par le sourire adressé par Augustin à sa mère, la maîtrise du discours est confiée à Monique, avec l'assentiment de celui qui a invité et reçoit. D'ordinaire, c'est le maître du discours qui pose les questions et tire les conclusions. Or, dans ce paragraphe, c'est Monique qui conclut la discussion, qui se clôt par les rires, par une formulation très énigmatique et difficilement interprétable. En effet, Monique emploie le terme caducarii, expression familière pour désigner ceux qui souffrent d'épilepsie, pour qualifier les académiciens, qui recherchent la sagesse tout en pensant ne jamais pouvoir l'obtenir. Plus loin dans le dialogue, Navigius, l'autre fils de Monique, revient sur cette expression, qu'il juge très familière et la justifie par le fait que, comme les épileptiques, les académiciens sont dans les ténèbres ${ }^{39}$. Mais l'explication est retardée et reste cependant très énigmatique ${ }^{40}$. L'entretien se conclut sur cette formule mystérieuse et sur le rire des convives ${ }^{41}$. Pour comprendre ce dernier, il est nécessaire de se rappeler le cadre des discours, il s'agit d'une discussion philosophique lors d'un banquet; la joie, la bonne humeur y sont donc de circonstance ${ }^{42}$. De plus l'attitude de Monique qui lâche une 
formule énigmatique avant de se retirer n'est pas sans faire penser à Diogène le Cynique qui se lève pour démontrer à Zénon que le mouvement existe, ce dont Augustin se souvient dans le De ordine.

En effet, le troisième entretien du De ordine s'achève également sur un mot d'esprit ; alors que la communauté s'apprêtait à parler du mouvement, un serviteur vient annoncer que le repas est prêt et Augustin affirme : « ce qu'est le mouvement, ce serviteur nous oblige non à le définir, mais à le faire voir à nous-mêmes ». Ce bon mot provoque le rire et le départ des convives ${ }^{43}$. Les deux personnages, Monique et Augustin, fonctionnent donc de façon, sinon identique, du moins parallèle, d'autant plus qu'Augustin présente sa mère comme un maitre de philosophie, comme nous l'avons vu plus haut; Augustin va même jusqu'à affirmer que lui, maître du dialogue, pourrait devenir son disciple ; ce que nous nous proposons d'examiner dans un dernier temps.

\section{Monique, une chrétienne, guide et médiatrice}

\section{Monique, modèle de la philosophie : quand une mère dépasse le maître}

Dans le De ordine, Augustin explique, ce qui pourrait surprendre son lecteur, pourquoi, bien que les Écritures condamnent la philosophie, Monique est philosophe.

Nunc uero cum eam multo plus quam meipsum diligas, et nouerim quantum me diligas, cumque in ea tantum profeceris, ut iam nec cuiusuis incommodi fortuiti nec ipsius mortis, quod uiris doctissimis difficillimum est, horrore terrearis, quam summam philosophiae arcem omnes esse confitentur, egone me non libenter tibi etiam discipulum dabo?

(De ordine, I, 32, BA 4/2, p. 156)

Comme tu aimes la sagesse bien plus que moi et que je sais combien tu m'aimes, et comme tu as fait à son sujet des progrès tels que tu n'es plus effrayée par la crainte de quelque malheur fortuit ni de la mort même, ce qui est la chose la plus difficile pour les hommes les plus savants, car c'est de l'aveu de tous la cime de la philosophie, est-ce que moi, je n'aimerai pas me confier à toi comme ton disciple?

De façon surprenante, le maître de parole qu'est Augustin reconnaît qu'il est redevable d'une femme sans culture qui est sa mère ; c'est elle la philosophe et lui le disciple. En fait, Monique aime la sagesse, parce qu'elle est chrétienne ; aimer la sagesse, pour Augustin, c'est aimer Dieu ou le Christ. Mais Augustin n'explicite pas encore ce point $^{44}$; la dimension chrétienne n'est pas ici précisée. La conversation philosophique est ajournée sur cet aveu ${ }^{45}$. L'entretien se clôt donc sur un énoncé surprenant, Monique, une femme, sans réelle culture, serait une philosophe dont Augustin voudrait se constituer le disciple. Comment le lecteur auquel s'adresse Augustin peut-il ne pas être surpris par un tel propos?

\section{À travers Monique, la voix de Dieu}

21 Monique est en réalité une vraie philosophe, parce, comme tout être humain doué de raison, Dieu est présent en elle. Dans le De beata uita, après une première question sur le bonheur à laquelle tous répondent d'une voix unanime, Monique répond seule à une seconde question de son fils. Ce dernier « sourit alors dans un transport de joie " $^{46}$ et dit :

Ipsam, inquam, prorsus, mater, arcem philosophiae tenuisti. Nam tibi procul dubio uerba defuerunt, ut non sicut Tullius te modo panderes, cuius de hac sententia uerba ista sunt. Nam in Hortensio, quem de laude ac defensione philosophiae librum fecit (...).

(De beata uita, 2, 10, BA 4/1, p. 72) 
Absolument, c'est le sommet même de la philosophie que tu as occupé. Car il n'y a pas de doute, ce sont les mots qui t'ont manqué pour t'empêcher, il y a un instant ${ }^{47}$ de donner libre cours à ta pensée, comme le fait Tullius, qui a les formules sur ce thème : c'est dans l'Hortensius, un livre qu'il a consacré à la gloire et à la défense de la philosophie (...).

Augustin approuve ici sa mère, par des propos très élogieux, puisqu'il met en parallèle sa mère avec le protreptique de Cicéron, qui a eu un rôle considérable dans l'itinéraire spirituel d'Augustin. De même que Cicéron, Monique peut apparaître comme le défenseur de la philosophie, mais sans toutefois connaître cet auteur, ni son œuvre. Par ailleurs, Augustin souligne le progrès accompli par Monique qui, dans un premier temps, n'a pas pleinement convaincu, alors qu'après sa deuxième intervention et le commentaire d'Augustin, l'auditoire lui est entièrement favorable :

In quibus uerbis illa sic exclamabat, ut obliti penitus sexus eius, magnum aliquem uirum considere nobiscum crederemus, me interim, quantum poteram, intellegente ex quo illa et quam diuino fonte manarent.

(De beata uita, 2, 10, BA 4/1, p. 72)

Elle s'exclama d'une telle manière, qu'oubliant tout à fait son sexe, nous avions l'impression que quelque grand homme siégeait parmi nous, cependant que je comprenais, dans la mesure où je le pouvais, de quelle source - et combien divine provenaient ses exclamations.

Reprenant des expressions appliquées à Platon par Cicéron dans les Tusculanes ${ }^{48}$, Augustin explique que Monique, par son discours, n'est plus une femme, mais est devenue un homme, la sagesse étant l'apanage de la gent masculine, notamment pour le public lettré auquel s'adresse Augustin et qui est mis en scène en partie dans le dialogue. La précision donnée ensuite par Augustin est fondamentale ; elle va au-delà d'une opposition hommefemme : à travers Monique, c'est Dieu qui s'exprime ${ }^{49}$. On peut d'ailleurs noter que la correction apportée par Augustin, «dans la mesure où je le pouvais ", est l'un des rares éléments caractéristiques des interventions de Monique, qui précise souvent qu'elle parle comme elle le peut ${ }^{50}$. La précision se comprend mieux si l'on se rappelle qu'Augustin n'est pas encore baptisé, il n'a pas reçu la grâce du baptême. Il saisit donc imparfaitement quelle source peut s'exprimer à travers la bouche de sa mère qui, elle, est baptisée.

Dans le monologue final d'Augustin dans le De beata uita, ponctué par les sourires de tous ${ }^{51}$ , Monique n'est pas entièrement convaincue par la démonstration de son fils et établit un parallèle entre indigence et malheur, en s'appuyant sur un exemple extrait de l'Hortensius de Cicéron, que vient de donner Augustin :

Ubi cum omnes mirando exclamassent, me ipso etiam non mediocriter alacri atque laeto, quod ab ea potissimum dictum esset quod pro magno de philosophorum libris atque ultimum proferre paraueram: uidetisne, inquam, aliud esse multas uariasque doctrinas, aliud animum attentissimum in Deum ? Nam unde ista quae miramur, nisi inde procedunt?

(De beata uita, 4, 27, BA 4/1, p. 110)

À cet endroit, tous se récrièrent d'admiration et, moi aussi, je n'étais pas peu gai et réjoui de voir que c'était elle qui, en priorité, avait énoncé ce que je m'étais préparé pour présenter comme une chose importante extraite des écrits des philosophes et à faire venir en dernier lieu. Voyez-vous, dis-je, que des doctrines multiples et diverses sont une chose et un esprit très attentif à Dieu une autre chose ? Car ces paroles que nous admirons, d'où procèdent-elles, sinon de cette source?

Augustin confirme ici ce qu'il affirmait plus haut: ce qui est admirable chez Monique, c'est qu'elle est le véhicule de la parole de Dieu. Or, Augustin clôt le dialogue en expliquant que la source de la sagesse est Dieu qui, par la raison, est présent en l'homme 
créé à son image. Ce dernier doit alors retourner à cette source, au Dieu unique, à la substance unique ${ }^{52}$. Monique réagit :

Hic mater recognitis uerbis quae suae memoriae penitus inhaerebant, et quasi euigilans in fidem suam, uersum illum sacerdotis nostri : " foue precantes, Trinitas », laeta effudit, atque subiecit: "haec est nullo ambigente beata uita, quae uita perfecta est, ad quam nos festinantes posse perduci, solida fide, alacri spe, flagrante caritate praesumendum est ».

(De beata uita, 4, 35, BA 4/1, p. 126)

À ce moment là, ma mère, reconnaissant les mots qui s'étaient profondément gravés en sa mémoire et s'éveillant en quelque sorte à sa foi, joyeuse, laissa tomber ce vers de notre évêque : «Protège, Trinité, ceux qui te prient ! $»^{53}$ et elle ajouta : « voilà, nul n'en doute, ce qu'est la vie heureuse, qui est la vie parfaite à laquelle une foi solide, une espérance allègre, une charité brûlante doivent nous faire présumer que nous pourrons être amenés rapidement ».

Le dialogue se clôt ainsi. L'intervention de Monique est donc la dernière parole significative du dialogue. Elle illustre la théorie chrétienne de la réminiscence, de l'illumination, qu'Augustin développera davantage dans le De magistro. Cette intervention est construite sur un schéma proche de la clôture du premier entretien : le motif de la joie et une formule frappante, ici extraite d'une hymne d'Ambroise, énoncée de façon rapide et présentée comme spontanée (effudit). De plus, le dialogue se conclut par une évocation de l'évêque de Milan, Ambroise, qui donnera le baptême à Augustin. Monique qui, parce qu'elle est une femme et n'est pas lettrée, semblait le plus étrangère au dialogue, se révèle être la véritable philosophe, car elle est chrétienne. Le duo que forment Augustin et sa mère apparaît en pleine lumière : la discussion avec Augustin permet à Monique de s'interroger, de chercher la vérité en elle-même, comme l'esclave dans le Ménon; parce qu'elle est baptisée, elle est comme supérieure à son fils, qui ne l'est pas encore, et conclut ainsi la discussion.

De même, à la fin du De ordine, Augustin conclut l'entretien en demandant à Monique de prier Dieu, afin que ses amis et lui puissent progresser dans la recherche de la sagesse.

Oremus ergo, (...) ut ea proueniant, quae nos bonos faciant ac beatos. Quae uota ut deuotissime impleantur, tibi maxime hoc negotium, mater, iniungimus, cuius precibus indubitanter credo atque confirmo mihi istam mentem Deum dedisse, ut inueniendae ueritati nihil omnino praeponam, nihil aliud uelim nihil cogitem, nihil amen. Nec desino credere nos hoc tantum bonum, quod te promerente concupiuimus, eadem te petente adepturos.

(De ordine, II, 52, BA 4/2, p. 320)

Il faut prier pour que (...) Dieu accorde les choses qui rendent bons et heureux. Faire que ces demandes soient accomplies dans la plus grande piété, c'est la tâche dont nous te chargeons avant tout, ma mère, toi dont les prières, je le crois et l'assure sans hésiter, m'ont inspiré cet état d'esprit de ne préférer absolument rien à la découverte de la vérité, de ne rien vouloir, penser, aimer d'autre, et je ne cesse de croire que ce bien si grand, auquel tu nous as fait aspirer par ton mérite, nous allons l'obtenir aussi par tes demandes.

Monique, second maître de parole au cours du dialogue, est donc présentée comme un guide et un modèle ${ }^{54}$. Car elle possède sa philosophie, qui est le christianisme, elle doit faire en sorte que les protagonistes du dialogue connaissent le bonheur qu'apporte la foi. Ce qui différencie ainsi Monique du reste du groupe, et qui justifie sa présence au sein du dialogue, est le fait qu'elle soit déjà baptisée, alors que les autres attendent le baptême. Elle peut donc, à ce titre, être considérée comme un modèle. C'est pour cela qu'Augustin fait d'elle un second maître de parole. Ainsi, son statut de femme, mais plus encore de femme qui n'est pas lettrée n'est mis en valeur que pour mieux renforcer la supériorité, 
paradoxale, que lui donne le baptême et, par conséquent, le pouvoir de Dieu en l'homme. Comme Augustin le rappelle à la fin de sa vie dans la lettre $2^{*}$, adressée au catéchumène Firmus, dont l'épouse est baptisée, une femme, inférieure, notamment par sa culture, à l'homme, si elle est baptisée, est supérieure même à un homme lettré qui est catéchumène, car Firmus pourra lui apprendre ce qu'il a lu, mais ce qu'elle sait par le sacrement du baptême, elle ne pourra pas le révéler ${ }^{55}$.

\section{BIBLIOGRAPHIE}

BASSI D. (1930), « Monica nei dialoghi di Casiciaco », dans Augustiniana nel XV centenario dalla morte di sant'Agostino. 430-1930, Naples, p. 51-61.

BROWN P. (2001), La vie de saint Augustin, Paris.

CAMPS G. (1992), L’Afrique du Nord au féminin. Hérö̈nes du Mahgreb et du Sahara, Paris, p. 92-108.

CONYBEARE C. (2006), The irrational Augustine, Oxford.

COURCELLES P. (1943), Les lettres grecques en Occident. De Macrobe à Cassiodore, Paris.

COURCELles P. (1968), Recherches sur les Confessions de saint Augustin, Paris.

DoIGNON J. (1987), « Le De ordine, son déroulement, ses thèmes », dans L'opera letteraria di Agostino tra Cassicicum e Milano, Palerme, p. 113-150.

DJUTH M. (2008), « Augustine, Monica, and the Love of Wisdom », Augustinian Studies 39, p. $237-252$.

FRAZIER F. (1998), « Théorie et pratique de la paidia symposiaque dans les Propos de table de Plutarque ", dans M. Trédé et P. Hoffmann éd., avec la coll. de C. Auvray-Assayas, Le rire des anciens, Paris, p. 281-292.

Holte R. (1994), « Monica, “the philosopher” ", Augustinus 39, p. 293-316.

HOFFMANN M. (1966), Der Dialog bei den christlichen Schrifstellern der ersten vier Jahrhunderte, Berlin. LAMIRANDE E (1989), « Quand Monique, la mère d'Augustin, prend la parole », dans A. Zumkeller éd, Signum pietatis, Festgabe für Cornelius Petrus Mayer OSA zum 60. Geburtstag, Würzburg, p. 3-19.

LANCEL S. (1999), Saint Augustin, Paris.

MADEC G. (1996), Saint Augustin et la philosophie. Notes critiques, Paris.

MANDouze A. (1969), « Monique à Cassiciacum », Revue des études latines 47, 1969, p. 131-141.

MORE O'FERRALL M. (1975), « Monica, the mother of Augustine », Recherches augustiniennes 10, p. 23-43.

PIETRI C. (2000), art. «Flauius Mallius Theodorus 3 », dans Prosopographie chrétienne du Bas-Empire 2, Rome, p. 2167-2168.

PIZzolato F. (1987), « Il De beata vita o la possibile felicità nel tempo », dans L'opera letteraria di Agostino tra Cassicicum e Milano, Palerme. 
SAGINARIO G. (2005), Monica mia madre. Biografia critica della madre di Agostino, Rome.

voss B. R (1970), Der Dialog in der frühchristlichen Literatur, Munich.

\section{NOTES}

1. Je remercie vivement Jean-François Cottier et Anne-Isabelle Bouton-Touboulic pour leur relecture et leurs précieux conseils.

2. Confessions, VIII, 12, 29, BA 14, p. 66.

3. Confessions, IX, 2, 4, BA 14, p. 76.

4. Confessions, VIII, 6, 13, BA 14, p. 34 .

5. De beata uita, 1, 6, BA 4/1, p. 63.

6. Confessions, IX, 4, 8, BA 4/1, p. 84.

7. Sur la retraite d'Augustin à Cassiciacum, voir BROWN 2001, p. 145-162 ; LANCEL 1999, p. 146-162.

8. Pour une présentation rapide de Monique, voir BROWN 2001, p. 31-39; LANCEL 1999, p. 24-30.

9. Contra academicos, II, 5, 13 ; comme le souligne A. MANDOUZE 1969, p. 132.

10. Voir notamment LAMIRANDE 1989, p. 3-19 ; MORE O'FERRALL 1975, p. 23-43 ou, plus récemment, SAGINARIO 2005.

11. Pour une étude d'ensemble du dialogue, voir PIzzolato 1987, p. 31-112. Nous utiliserons Augustin, La vie heureuse, introd., éd., trad. et notes par J. Doignon, Paris, Bibliothèques Augustiniennes 4/1, 1986.

12. Pour une étude d'ensemble de ce texte, voir DoIGNON 1987, p.113-150. Nous utiliserons Augustin, L'ordre, introd., éd., trad. et notes par J. Doignon, Paris, Bibliothèques Augustiniennes 4/2, 1997.

13. Voir MANDOUZE 1969, p. 131-141 ; CAMPS 1992, p. 92-108 ; HOLTE 1994, p. 293-316 ; DJUTH 2008, p. 237-252.

14. C. CONYBEARE 2006, p. 59 sqq. propose une analyse de cette mise en scène, sur laquelle nous reviendrons au cours de notre réflexion.

15. Sur cette question, voir l'analyse de G. MADEC 1996, p. 45-52.

16. Voir PIETRI 2000, p. 2167-2168 ; COURCELLES 1943, p. 122-128 et COURCELLES 1969, p. 153-156.

17. Comme le souligne B. R. voss 1970, p. 210.

18. Voir C. PIETRI, art. «Zenobius 1 », dans Prosopographie chrétienne du Bas-Empire, 2, Rome, 2000, p. 2378.

19. De ordine, I, 29, BA 4/2, p. 142.

20. Diotime n'est jamais évoquée par Cicéron.

21. Comme le souligne D. BASSI 1930, p. 53.

22. Sur ce point, nous nous opposons à l'analyse de C. CONYBEARE (2006, p. 92), qui estime que la présence de Monique est importante, non pas tant par son manque d'éducation, relatif, que par son sexe. L'analyse des textes, cependant, montre que l'absence de culture est fondamentale pour comprendre la figure de Monique.

23. CONYBEARE 2006, p. 105 sqq. se demande, sans trouver de solution, à quelle femme peut songer Augustin.

24. Comme le soulignent M. HOfFMAnN 1966, p. 143 sqq. et B. R. voss 1970, p. 209.

25. Comme le note C. CONYBEARE 2006, p. 74.

26. De ordine, II, 22, BA 4/2, p. 226.

27. De ordine, I, 29, BA 4/2, p. 142-144.

28. De ordine, I, 30, BA 4/2, p. 144-146.

29. De beata uita, 3, 19, BA 4/1, p. 94. 
30. Comme le souligne A. MANDouze 1969, p. 136 ; cf. De ordine, II, 23, BA 4/2, p. 230-232.

31. De beata uita, 4, 23, BA 4/1, p. 100.

32. Ce point semble ne pas avoir été suffisamment souligné par C. CONYBEARE 2006.

33. De beata uita, $2,7, B A 4 / 1$, p. 64 .

34. De beata uita, 2, 8, BA 4/1, p. 66 .

35. De beata uita, 2, 11, BA 4/1, p. 76 .

36. De beata uita, 2, 12, BA 4/1, p. 78 .

37. De beata uita, 2, 12, BA 4/1, p. 78.

38. De beata uita, 3, 18, BA 4/1, p. 90-92.

39. De beata uita, 3, 20, BA 4/1, p. 94.

40. Il reste très difficile de trouver une explication satisfaisante à cette expression, d'autant plus qu'il s'agit, dans cette acception, d'un néologisme, qui n'est pas le mot traditionnellement utilisé pour désigner l'épilepsie. On pourrait penser que l'image de l'épileptique qui tombe (comme le montre la racine du mot caducarii, caduc-) se comprend si l'on se souvient que la religion chrétienne est un chemin, une uia (cf. Jean, 14, 6: je suis le chemin, la vérité et la vie). Or l'académicien, qui considère que l'on ne peut atteindre la vérité et la sagesse, ne parvient pas à connaître le Christ, qui est le chemin et la vérité, et par conséquent tombe.

41. Contrairement à C. CONYBEARE (2006, p. 79), nous ne pensons pas que le rire dans le dialogue soit un simple élément du décor ou qu'il crée simplement une pause dans le dialogue. Ce motif, qui appartient, certes, à la littérature symposiaque (comme le souligne FRAZIER 1998, p. 281-292), auquel le De beata uita peut appartenir, permet ici de souligner l'incapacité de la raison des académiciens.

42. Cf. Minucius Felix, Octauius, 40,4 .

43. De ordine, II, 18, BA 4/2, p. 216.

44. Sur cet aspect, voir DJUTH 2008, p. 242.

45. De ordine, I, 33, BA 4/2, p. 158.

46. De beata uita, 2, 10, BA 4/1, p. 70 .

47. De beata uita, 2, 8, BA 4/1, p. 66-68.

48. $\mathrm{V}, 12,36$ : sancto augustoque fonte... manabit oratio. $B A 4 / 1$, p. 73, n. 36 .

49. Cf. Épitre aux Galates, 3, 28 : « il n'y a ni Juif, ni Grec, il n'y a ni esclave ni homme libre, il n'y a ni homme ni femme ; car tous vous ne faites qu'un dans le Christ Jésus ».

50. De beata uita, 3, 21, BA 4/1, p. 96 ; comme le souligne C. CONYBEARE 2006, p. 82.

51. De beata uita, 4, 27, BA 4/1, p. 108-110.

52. De beata uita, 4, 35, BA 4/1, p. 126.

53. Ambroise, Hymne, 2 (Deus creator omnium), 32.

54. Nous ne pensons pas, comme le fait M. DJUTH (2008, p. 246), que Monique est le réel médiateur des dialogues et qu'Augustin est relayé au second plan, car c'est bien le fils qui distribue la parole, qui reprend les propos de sa mère et les développe dans les longs monologues.

55. Lettre, $2^{*}, 4$, BA 46B, p. 66.

\section{RÉSUMÉS}

Dans deux dialogues philosophiques, le De beata uita et le De ordine, rédigés avant qu'il ne reçoive le baptême, Augustin met en scène sa mère, Monique. Cette dernière dialogue et philosophe avec 
les autres participants, qui sont des hommes. Cette prise de parole, d'une femme sans culture, n'allait pas de soi à cause du genre, un dialogue philosophique, des dédicataires, ainsi que du lectorat visé ; par conséquent, Augustin la justifie. Deux éléments caractérisent les interventions de Monique : leur liberté et leur effet dans le débat. Monique et Augustin, maître du discours, forment un duo. Augustin va même jusqu'à affirmer qu'il pourrait se faire son disciple. Ce qui différencie Monique du reste du groupe est le fait qu'elle soit déjà baptisée, alors que les autres attendent le baptême.

In two philosophical dialogues, the De beata uita and the De ordine, written by Augustine before he received baptism, Augustine uses his mother, Monica, as a character, who takes part to the dialogue and philosophizes with the other participants, who are men. A woman speaking, specially a woman without culture, was unusual because of the genre, a philosophical dialogue, of the dedicatees, and of the reader Augustine has in mind ; in fact, he justifies her presence in the dialogues. The words of Monica are free and have a real impact on the discussion. Monica seems to play a duet with Augustine, the master of the dialogue. Augustine says he could become the disciple of his mother. In fact, the difference between Monica and the others is that she is baptized, whereas the others are waiting for baptism.

\section{INDEX}

Mots-clés : Augustin, femme, Monique, philosophie

Keywords : Augustine, Monica, philosophy, woman

\section{AUTEUR}

\section{MICKAËL RIBREAU}

Université Sorbonne Nouvelle - Paris 3 ; Centre d'Études Sur l'Antiquité Rémanente (EA 173) ; Institut d'É tudes Augustiniennes (UMR 8584) 